\title{
PRESENCIA DE SIINTOMAS ASOCIADOS A ENFERMEDADES ALÉRICAS POR CONSUMO DE ALIMENTOS EN NIÑOS ANTES Y DESPUÉS DE LOS SEIS MESES DE EDAD EN BARRANQUILLA - COLOMBIA
}

\section{PRESENCE OF SYMPTOMS ASSOCIATED WITH ALLERGIC DISEASES BY CONSUMPTION OF FOODS IN CHILDREN'S BEFORE AND AFTER OF SIX MONTHS OLD IN BARRANQUILLA - COLOMBIA}

\author{
Karol Cervantes De La Torre ${ }^{1}$, Francisco Guillen Grima ${ }^{2}$, Inés Aguinaga Ontoso ${ }^{3}$
}

\begin{abstract}
${ }^{1}$ Doctor en salud pública, Docente jornada laboral completa. Universidad Libre Barranquilla, Colombia, e-mail: karold.cervantesd@unilibre.edu.co; ${ }^{2}$ Doctor en Medicina, Departamento de Ciencias de la Salud. Universidad Pública de Navarra, Pamplona, IDISNA (Instituto de Investigación Sanitaria de Navarra), Pamplona, Medicina Preventiva, Clínica Universidad de Navarra, Pamplona, e-mail: f.guillen.grima@unavarra.es; ${ }^{3}$ Doctor en medicina y cirugía, Departamento de Ciencias de la Salud. Universidad Pública de Navarra, e-mail: ines.aguinaga@unavarra.es
\end{abstract}

Rev. U.D.C.A Act. \& Div. Cient. 20(2): 241-246, Julio-Diciembre, 2017

\section{RESUMEN}

En este trabajo de investigación, se determinó si la presencia de síntomas asociados a enfermedades alérgicas presenta relación con el consumo de alimentos en niños, antes y después de los 6 meses de edad, considerando estudios que indican que la ingesta temprana de alimentos, por parte de los menores, puede ser factor predisponente a padecimiento de enfermedades alérgicas. Se realizó mediante estudio de carácter descriptivo modalidad Cross, seccional en escuelas públicas y privadas del Distrito de Barranquilla, a nivel de básica primaria, aplicando el instrumento cuestionario ISAAC. Se obtuvieron 1.520 encuestas contestadas por los padres de los menores de niños y niñas, entre 6 y 7 años, $47 \%$ varones y $53 \%$ niñas. De los alimentos objetos del presente estudio, huevos, pescado y leche de vaca, los que presentaron valores significativos en relación a la presencia de síntomas enfermedades alérgicas fue: el pescado, para el asma, con un $p$ valor $<0,05$, y un OR: 2,40; en los demás alimentos, no se pudo establecer, mediante significancia relación entre el padecimiento de alergias y su consumo. Actualmente, no hay un consenso universal y este estudio tampoco concluye si la ablactación temprana y la ingesta de alimentos, antes de los seis meses de edad, pueda provocar la presencia de síntomas de enfermedades alérgicas. Se recomienda continuar con las recomendaciones de la Organización Mundial de la Salud e iniciar ingreso de alimentos a la dieta del menor, después de los seis meses.
Palabras clave: Alergia, Rinitis, Asma, Eccema, Alimentos.

\section{SUMMARY}

In this research, it was determined whether the presence of symptoms associated with allergic diseases is related to food consumption in children before and after 6 months of age, considering studies that indicate that the early intake of food by children Can be a predisposing factor to allergic diseases, was carried out by means of a descriptive cross sectional modality in public and private schools of the District of Barranquilla at primary level through the instrument ISAAC questionnaire. We obtained 1520 surveys answered by the parents of the minors of children between 6 and 7 years old, $47 \%$ male and $53 \%$ female. Of the food items of the present study, eggs, fish and cow's milk, those presenting significant values in relation to the presence of allergic diseases symptoms were: fish for asthma with a $p$ value $<0.05$, and an OR: 2.40 in other foods could not be established by a significant relationship between the allergy condition and its consumption. There is currently no universal consensus and this study also does not conclude whether early ablation and food intake before 6 months of age may lead to the presence of allergic disease symptoms, it is recommended to continue the recommendations of the World Health Organization and initiate food intake into the child's diet after six months.

Key words: Allergy, Rhinitis, Asthma, Eczema, Food. 


\section{INTRODUCCIÓN}

Los menores que presentan alergias son un tema de suma importancia para investigadores y personal clínico, por ser estas enfermedades incapacitantes de alto costo económico y estar en aumento, a lo largo del tiempo (Kay, 2001; Gupta et al. 2007). Se considera que los acelerados ritmos de vida junto a otros cambios medio ambientales, asociados a procesos industrializados, favorecen su aparición (Min Salud Chile, 2010). Los estudios realizados muestran que en países desarrollados, se presentan una mayor prevalencia en lo que concierne a este tipo de enfermedades, considerándose que, en gran medida, se debe a una fuerte preferencia por la dieta Fast Foods o como es conocida en entornos de habla hispana, comida rápida o comida procesada; se considera, que se da también, debido a una mayor contaminación del aire, por las fuertes emisiones de gases industriales y por emisiones del parque automotor (Beasley, 1998).

Una saludable alimentación regularmente entre el primer mes y los primeros años de edad no solo es relevante para el buen desarrollo de los sistemas y de los órganos del cuerpo humano (Amarasekera et al. 2013), sino que, además, se puede convertir en un factor protector para prevenirlas, aunque en revisiones sobre este tema, se han obtenido resultados heterogéneos (Tarini et al. 2006). Para identificar los factores que favorecen este fenómeno se ha empleado en 56 países el estudio ISAAC (International Study of Asthma and Allergies in Childhood), que ayuda a obtener posibles indicadores causales o desencadenantes de alergias, entre esos, el consumo de alimentos de la madre durante el embarazo y la ingesta de alimentos del menor antes y después de los seis meses de vida. En referencia a los alimentos, la ablactación antes de los cuatro meses y la ingesta de alimentos procesados antes de los seis meses, se encuentran ligados a la manifestación de alergias, como lo son el asma, la rinitis y el eccema (Kull et al. 2002). En un afán de hallar porqué se brindan alimentos que difieren de la leche materna a menores de 6 meses, se ha encontrado que depende, en gran medida, a factores geográficos, en lo que corresponde a la facilidad de adquisición de éstos y factores culturales, lo cual, es relevante para que el menor posea una dieta saludable, que contribuya a su buen desarrollo. Entre más tarde sea la ingesta de alimentos procesados a la dieta del menor, éste tendrá una mejor respuesta modular inmune, debido a que contó con el tiempo necesario para permitir la maduración de su sistema digestivo (Fallani et al. 2010).

El objetivo en el estudio fue correlacionar la presencia de alergias y el consumo de ciertos alimentos antes y después de los seis meses de edad, mediante preguntas contempladas en el cuestionario ISAAC, orientadas a los cuidadores o padres de los niños y niñas, residentes en Barranquilla, Colombia.

\section{MATERIALES Y MÉTODOS}

Se llevó a cabo un estudio descriptivo modalidad Cross Sectional, a 1.520 menores, matriculados en los planteles educativos del Distrito de Barranquilla y su área metropolitana, durante el periodo 2014 - 2015, para determinar la presencia de enfermedades alérgicas que ellos pudieran presentar por haber consumido alimentos antes y después de los seis meses de edad, utilizando la metodología ISAAC. Se tomaron, para este estudio, las preguntas relacionadas con el consumo de alimentos, como el huevo, la leche de vaca y el pescado antes y después de los 6 meses y la asociación de éstos con la aparición de enfermedades alérgicas, específicamente, asma, rinitis y eccema. Los datos fueron obtenidos a través de la madre, el padre o el responsable de la custodia, quienes, previamente, firmaron el consentimiento informado. Se realiza la validación de la fiabilidad del cuestionario, mediante el cálculo del Alfa de Cronbach, para las variables que presentan la misma escala.

A cada pregunta, se le aplicó la prueba, la hipótesis se plantean como se muestra a continuación y se utiliza el valor $p$, como criterio de decisión, para rechazar o no rechazar la hipótesis, de que las variables son independientes entre sí. Nivel de significancia: 0,05.

Para la selección de los colegios oficiales, se tomó la base de datos disponible de la Secretaría de Educación de Barranquilla (2011) y para los colegios privados, el listado de establecimientos educativos no oficiales del Ministerio de Educación Nacional, que presentaron pruebas saber en el periodo 2014. En la selección de los estudiantes, se tuvo en cuenta que los participantes cumplieran con el criterio del cuestionario ISAAC, en cuanto a edad.

Análisis estadístico: Para evaluar la significación de las diferencias, se estableció una P significativa por debajo de 0,05 . Se empleó un paquete estadístico SPSS V24 y se realizó el cálculo del Odds Ratio.

Consideraciones éticas: Se solicitó autorización a la dirección de cada uno de los planteles educativos, garantizándose que el nombre de los mismos no aparecería relacionado en los resultados del estudio; asimismo, se dispone del consentimiento informado, diligenciado por el responsable del menor.

\section{RESULTADOS Y DISCUSIÓN}

Se aplicaron 1.520 encuestas para ser diligenciadas por los padres o por los responsables legales del menor, adscritos a los planteles educativos. El valor de Alfa de Cronbach es obtenido para las preguntas de una misma medida fue 0,78 , el cual, se registra en un nivel aceptable, implicando que la 
escala utilizada fue apropiada para el estudio, garantizando fiabilidad en la prueba.

A la pregunta si ha tenido alguna enfermedad alérgica, como asma, rinitis o eccema, teniendo en cuenta el consumo de huevo antes y después de los 6 meses de edad del menor, se observa en la tabla 1 que, para el asma, las variables no están relacionadas entre sí, obteniéndose un valor $\mathrm{P}>0,05$, un OR: 1,0 lo que significa que la frecuencia del consumo de huevo antes y después de los 6 meses no está relacionado con la presencia de asma. Para la rinitis, el valor obtenido OR fue de: 0.79 y una $\mathrm{P}>0,05$, pudiendo concluir que la frecuencia del consumo de huevo antes y después de los 6 meses no está relacionado con la presencia de rinitis; éste podría, incluso, actuar como factor protector. En el eccema, se obtuvo como resultado una $\mathrm{P}>0,05$, OR: 1,2, demostrando que, prácticamente, no hay relación entre el consumo de huevo antes y después de los seis meses y la presencia de síntomas de eccema.
Referente al consumo de pescado antes y después de los seis meses de edad, se observa en la tabla 2 , que el asma, en la prueba, arrojó un valor $\mathrm{P}<0,05$ y una OR: 2,40 , por lo tanto, se puede concluir, con un $95 \%$ de confianza, que las variables están relacionadas entre sí, sí afecta la frecuencia del consumo de pescado antes y después de los 6 meses, en la presencia de asma; para la rinitis, los resultados de P fueron > 0,05 y OR: 1,06, donde las variables no están relacionadas entre sí, la frecuencia del consumo de pescado, antes y después de los 6 meses, no está relacionado con la presencia de rinitis; en lo concerniente al eccema, se encontró dependencia entre las variables, con una $\mathrm{P}<0,05$ y un OR: 0,62 , indicando que el consumo de pescado puede ser factor protector para el eccema.

Para la pregunta sobre la presencia de síntomas de enfermedades alérgicas ante el consumo de leche de vaca antes y después de 6 meses, los resultados (Tabla 3), en la relación

Tabla 1. Asociación entre alergias y consumo de huevo.

\begin{tabular}{|c|c|c|c|}
\hline \multicolumn{4}{|c|}{ Pruebas teniendo en cuenta el consumo de HUEVO antes y después de los 6 meses } \\
\hline Alergia & Odds ratio (OR) & IC & P \\
\hline Asma & 1,00 & $\begin{array}{c}\text { IC } 95 \% \\
{[0,5 ; 3,8]}\end{array}$ & 0,572 \\
\hline Rinitis & 0,79 & $\begin{array}{c}\text { IC } 95 \% \\
{[0,4 ; 2,9]}\end{array}$ & 0,616 \\
\hline Eccema & 1,20 & $\begin{array}{c}\text { IC } 95 \% \\
{[0,6 ; 3,4]}\end{array}$ & 0,678 \\
\hline
\end{tabular}

Tabla 2. Asociación entre alergias y consumo de pescado.

\begin{tabular}{|c|c|c|c|}
\hline \multicolumn{3}{|c|}{ Pruebas teniendo en cuenta el consumo de PESCADO antes y después de los 6 meses } \\
\hline Alergia & Odds ratio (OR) & IC & P \\
\hline Asma & 2,40 & $\begin{array}{l}\text { IC } 95 \% \\
{[1,3 ; 5,8]}\end{array}$ & 0,036 \\
\hline Rinitis & 1,06 & $\begin{array}{l}\text { IC } 95 \% \\
{[0,8 ; 1,2]}\end{array}$ & 0,512 \\
\hline Eccema & 0,62 & $\begin{array}{c}\text { IC } 95 \% \\
{[0,3 ; 0,8]}\end{array}$ & 0,025 \\
\hline
\end{tabular}


al asma, fueron una $\mathrm{P}>0,05$ y OR: 1,2, variables son independientes, donde la frecuencia del consumo de leche de vaca, antes y después de los 6 meses, no está relacionado con la presencia de asma. Para la rinitis, el resultado fue una $P<0,05$, aceptando así la hipótesis nula, lo que indica que la rinitis no está relacionada con el consumo de leche de vaca y un OR: 0,69, indicando que puede ser un factor protector a padecer la enfermedad. Para el eccema tampoco se encontró relación entre las variables, con un valor $\mathrm{P}>0,05$, con un 95\% de confianza y OR: 0,95.
Dentro de los resultados más relevantes a observar en este estudio están el no haber encontrado relación de significancia estadística entre el consumo de huevo antes y después de los seis meses de edad y la presencia de síntomas asociados a alergias; este resultado es similar a un estudio realizado en Australia, donde en una muestra de 2.589 menores, se pudo concluir que, a pesar de ser introducido el alimento antes de los 6 meses, es probable que las alergias no se manifiesten, siendo posible que la manera de preparación influya; el huevo cocinado en agua es el que representa menos

Tabla 3. Asociación entre alergias y el consumo de leche de vaca.

\begin{tabular}{|c|c|c|c|}
\hline \multicolumn{4}{|c|}{ Pruebas teniendo en cuenta el consumo de LECHE DE VACA antes y después de los 6 meses } \\
\hline Alergia & $\begin{array}{c}\text { Odds ratio } \\
(\text { OR })\end{array}$ & IC & P \\
\hline Asma & 1,20 & $\begin{array}{c}\text { IC } 95 \% \\
{[0,9 ; 5,2]}\end{array}$ & 0,692 \\
\hline Rinitis & 0,69 & $\begin{array}{c}\text { IC } 95 \% \\
{[0,4 ; 0,9]}\end{array}$ & 0,040 \\
\hline Eccema & 0,95 & $\begin{array}{c}\text { IC } 95 \% \\
{[0,7 ; 10,2]}\end{array}$ & 0,568 \\
\hline
\end{tabular}

riesgo al considerarse que el hervor desnaturaliza las proteínas, debilitando su efecto alérgeno (Koplin et al. 2010). Es necesario presentar también investigaciones, donde se considera que la introducción temprana es predisponente a alergias (Kaplan et al. 2000) y se relaciona este tipo de alimentos, como factores predisponentes a alergias futuras, ya que se considera que se observan con una gran frecuencia en alimentos, como el huevo, los pescados, la leche de vaca y algunos cereales; asimismo, se ha encontrado relación entre el consumo temprano de huevo y el eccema y reducción del síntoma de picor, referido por el paciente, al dejar su consumo (Rojas \& Quezada, 2013). No se puede desconocer que otros factores, como los de orden social, entre esos los culturales, influyen en la manifestación de estas enfermedades, tópicos interesantes, que se recomiendan sean objeto de estudio en investigaciones futuras, ya que se consideran pueden influir notablemente en los resultados (Sánchez et al. 2014). Para los alimentos base de nuestro estudio y su asocio con enfermedades de base alérgica, la evidencia no es concluyente y existen estudios de revisiones sistemáticas, que han derivado en guías de recomendación sobre alimentación a lactantes, que muestran que, el consumo de ciertos alimentos, entre ellos el huevo, no sensibiliza (Romero et al. 2016). Ante la presencia de una posible alergia por alimentos, se debe verificar por pruebas, ya que se debe tener cuidado especial en retirar alimentos de la dieta de los menores, en etapa de crecimiento, por riesgo a desfavorecer su estado nutricional, aumentando la posibilidad de padecer enfermedades crónicas no transmisibles, a largo plazo (Greenhawt, 2010).

Este estudio presentó relación con la variable ingesta de pescado antes y después de los seis meses y el eccema, que se muestra como factor protector, siendo este hallazgo similar al encontrado en la literatura, que manifiesta que el consumo regular de pescado, después del año de vida e, incluso, posterior hasta los 4 años, disminuyen la manifestación de enfermedades (Morales et al. 2015); los pescados son fuente nutricional de ácidos omega 3, los cuales, favorecen el desarrollo de un óptimo sistema inmune, aunque, en este caso, también podemos encontrar evidencias contrarias en la literatura, que no respaldan el hallazgo de la investigación (Cattaneo et al. 2011). Resultados similares al del consumo de huevo presentó el consumo de leche de vaca antes y después de los seis meses de edad, no encontrando relación de esta con la presencia de síntomas asociados a asma, a rinitis y a eccema; este resultado es contradictorio a investigaciones realizadas, en donde su consumo temprano (antes de los seis meses de edad) puede ser considerado como factor predisponente a alergias (Prescott, 2003). Consideramos que nuestro hallazgo se puede deber que, al momento de contestar la pregunta y por la manera como está escrita, no 
se especifica si la fórmula de la leche contiene sustancias que le disminuyen altamente su potencial, como agente alérgeno, como proteínas de soya e hidrolizados de caseína, entre otros.

Bien valdría tener en cuenta, en futuras investigaciones, por los hallazgos encontrados, elementos que aún están siendo estudiados y que, al parecer, juegan un papel importante, en este tipo de enfermedades, como lo es la microbiota, a nivel intestinal (Navarro \& Araya, 2016), elemento que no fue abordado en este estudio. En cuanto a la edad adecuada para la introducción de la alimentación complementaria, entendida ésta, según criterio de la Organización Mundial de la Salud, como todo alimento diferente a la leche materna, a pesar que hay una tendencia que son los 6 meses de edad, este rango varía según el país, en Colombia, se recomienda que la introducción de semisólidos sea a partir de los 6 meses, pero se puede observar que, según las regiones, el acceso a los alimentos y la capacidad económica de las familias, se puede dar antes; en zonas costeras, como el caso de Barranquilla, los alimentos que presentan mayor acceso, en cuanto a disponibilidad, están los pescados y los mariscos, los cuales, son consumidos de forma frecuente. Se sugiere en el estudio, que este factor sea determinante para reducción del eccema, como enfermedad alérgica en los menores, que participaron en la investigación.

Estudios de este tipo brindan actualización, en lo que respecta a enfermedades que están en aumento y su relación con factores propios de la zona geográfica, como es el caso de la dieta alimenticia, ampliando así el marco etiológico y favoreciendo un tratamiento certero.

Teniendo en cuenta nuestros resultados y relacionándolos con la literatura consultada, no se puede establecer, de manera certera, una conclusión, que asevere que la introducción de ciertos alimentos antes de los 6 meses de edad no serán potencialmente alérgenos, por lo consiguiente, se recomienda den, de forma complementaria, después de los 6 meses de edad y, antes de esta edad, considerar, de manera exclusiva, la lactancia materna (Toca et al. 2015).

Conflicto de intereses: El manuscrito fue preparado y revisado con la participación de todos los autores, quienes declaramos que no existe conflicto de intereses, que ponga en riesgo la validez de los resultados presentados.

\section{BIBLIOGRAFÍA}

1. AMARASEKERA, M.; PRESCOTT, S.L.; PALMER, D.J. 2013. Nutrition in early life, immune programming and allergies: the role of epigenetics. Asian Pacific J. Allergy Immunology. 31(3):175-182.
2. BEASLEY, R. 1998. Worldwide variation in prevalence of symptoms of asthma, allergic rhinoconjuntivitis, and atopic eczema: ISAAC. Lancet. 351:1225-1232.

3. CATTANEO, A.; WILLIAMS, C.; PALLÁS, C.R.; HERNÁNDEZ- AGUILAR, M.T.; LASARTE-VELILLAS, J.; LANDA-RIVERA, L.; OUDESLUYS-MURPHY, A. 2011. ESPGHAN's 2008 recommendation for early introduction of complementary foods: how good is the evidence? Maternal and Child Nutrition. 7(4):335-343.

4. FALLANI, M.; YOUNG, D.; SCOTT, J.; NORIN, E.; AMARRI, S.; ADAN, R.; DORE, J. 2010. Intestinal microbiota of 6-week-old infants across Europe: geographic influence beyond delivery mode, breastfeeding, and antibiotics. Journal of Pediatric Gastroenterology Nutrition. 51(1):77-84.

5. GREENHAWT, M. 2010. The role of food allergy in atopic dermatitis. Allergy and Asthma Proceedings. 31:392397.

6. GUPTA, R.; SHEIKH, A.; STRACHAN, D.; ANDERSON, H. 2007. Time trends in allergic disorders in the UK. Thorax. 62(1):91-6.

7. KAPLAN, A.; BOUSQUET, J.; NASPITZ, C. 2000. Prevention of allergy and asthma. Allergy Clin. Immunol. Int. 12(6):288-299.

8. KAY, A.B. 2001. Allergy and Allergic Diseases. New England J. Medicine. 344:30-36.

9. KOPLIN, J.J.; OSBORNE, N.J.; WAKE, M.; MARTIN, P.E.; GURRIN, L.C.; ROBINSON, M.N.; TEY, D.; SLAA, M.; THIELE, L.; MILES, L.; ANDERSON, D.; TAN, T.; DANG, T.D.; HILL, D.; LOWE, A.J.; MATHESON, M.C.; PONSONBY, A.L.; TANG, M.L.; DHARMAGE, S.C.; ALLEN, K.J. 2010. Can early introduction of egg prevent egg allergy in infants? A population-based study. J Allergy Clin Immunol. 126:807-813.

10. KULL, I.; WICKMAN, M.; LILJA, G.; NORDVALL, S.L.; PERSHAGEN, G. 2002. Breastfeeding and allergic diseases in infants - a prospective birth cohort study. Archives of Disease Childhood. 87(6):478-481.

11. MINISTERIO DE EDUCACIÓN NACIONAL. 2014. Listado de Establecimientos educativos No Oficiales en Colombia. Disponible desde Internet en: http://www. mineducacion.gov.co/1621/articles-354560_Establecimientos_educativos_no_oficiales_que_superan_el_percentil_20_pdf (con acceso el 10/09/2014). 
12. MINISTERIO DE SALUD DE CHILE. 2010. Encuesta Nacional de Salud ENS Chile: 2009-2010. Disponible desde Internet en: http://web.minsal.cl/portal/url/ item/bcb03d7bc28b64dfe040010165012d23.pdf (con acceso el 14/11/2016).

13. MORALES, C.; BEDOLLA, M.; LÓPEZ, L.; ROMERO, C. 2015. Prevalencia de las enfermedades alérgicas y su asociación con la lactancia materna y el inicio de la alimentación complementaria en niños de edad escolar de Ciudad Guzmán, México. Archivos Argentinos de Pediatría. 113(4):324-330.

14. NAVARRO, E.; ARAYA, M. 2016. Inicio de alimentación complementaria y riesgo de enfermedad celíaca y alergia alimentaria: ¿De qué evidencia disponemos? Rev. Chilena de Nutrición. 43(3):315-320.

15. PRESCOTT, S.L. 2003. Early origins of allergic disease: a review of processes and influences during early immune development. Current opinion in allergy and clinical immunology. 3:125-132.

16. ROJAS, R.; QUEZADA, A. 2013. Relación entre dermatitis atópica y alergia alimentaria. Rev. Chilena de Pediatría. 84(4):438-450.

17. ROMERO, E.; VILLALPANDO, S.; PÉREZ, A.B.; IRECHETA, M.; ALONSO, C.G.; LÓPEZ, G.E.; GARCÍA, A.; OCHOA, E.; ZÁRATE, F.; LÓPEZ, G.T.; CHÁVEZ, C.; GUAJARDO, M.; VÁZQUEZ, S.; PINZÓN, B.A.; TORRES, K.N.; VIDAL, J.D.; MICHEL, P.L.; LÓPEZ, I.N.; ARROYO, L.V.; ALMADA, P.; SALTIGERAL, P.; RÍOS, A.; DOMÍNGUEZ, L.; RODRÍGUEZ, P.; CRABTREE,
Ú.; HERNÁNDEZ, V.; PINACHO, J.L. 2016. Consenso para las prácticas de alimentación complementaria en lactantes sanos. Bol Med Hosp Infant Mex. 73(5):338-356.

18. SÁNCHEZ, J.; RESTREPO, M.; MOPAN, J.; CHINCHILLA, C.; CARDONA, R. 2014. Alergia a la leche y al huevo: diagnóstico, manejo e implicaciones en América Latina. Biomédica. 34(1):143-156.

19. SECRETARÍA DE EDUCACIÓN DE BARRANQUILLA. 2011. Listado de Instituciones Educativas Oficiales. Disponible desde Internet en: http://www.sedbarranquilla.gov.co/sed/index.php?option $=$ com_conte nt $\&$ view $=$ article\&id $=2310$ ltemid $=46$ (con acceso 10/09/2014).

20. TARINI, B.; CARROLL, A.; SOX, C.; CHRISTAKIS, D. 2006. Systematic review of the relationship between early introduction of solid foods to infants and the development of allergic disease. Archives of Pediatrics Adolescent Medicine. 160(5):502-507.

21. TOCA, M.; TONIETTI, M.; VECCHIARELLI, C. 2015. Nutrición pre y posnatal: impacto a largo plazo en la salud. Archivos Argentinos de Pediatría. 113(3):254259.

Recibido: Febrero 28 de 2017

Aceptado: Septiembre 4 de 2017

Cómo citar:

Cervantes De La Torre, K.; Guillen Grima, F.; Aguinaga Ontoso, I. 2017. Presencia de síntomas asociados a enfermedades alérgicas por consumo de alimentos en niños antes y después de los seis meses de edad en Barranquilla - Colombia. Rev. U.D.C.A Act. \& Div. Cient. 20(2): 241-246. 